\title{
A BRUHAT ORDER FOR THE CLASS OF $(0,1)$-MATRICES WITH ROW SUM VECTOR R AND COLUMN SUM VECTOR * $^{*}$
}

\author{
RICHARD A. BRUALDI ${ }^{\dagger}$ AND SUK-GEUN HWANG ${ }^{\ddagger}$
}

\begin{abstract}
Generalizing the Bruhat order for permutations (so for permutation matrices), a Bruhat order is defined for the class of $m$ by $n(0,1)$-matrices with a given row and column sum vector. An algorithm is given for constructing a minimal matrix (with respect to the Bruhat order) in such a class. This algorithm simplifies in the case that the row and column sums are all equal to a constant $k$. When $k=2$ or $k=3$, all minimal matrices are determined. Examples are presented that suggest such a determination might be very difficult for $k \geq 4$.
\end{abstract}

Key words. Bruhat order, Row sum and column sum vectors, Interchanges, Minimal matrix.

AMS subject classifications. 05B20, 06A07, 15A36.

1. Introduction. Let $R=\left(r_{1}, r_{2}, \ldots, r_{m}\right)$ and $S=\left(s_{1}, s_{2}, \ldots, s_{n}\right)$ be nonincreasing, positive integral vectors, so that

$$
r_{1} \geq r_{2} \geq \cdots \geq r_{m}>0 \quad \text { and } \quad s_{1} \geq s_{2} \geq \cdots \geq s_{n}>0 .
$$

Then $\mathcal{A}(R, S)$ denotes the class of all $m$ by $n(0,1)$-matrices with row sum vector $R$ and column sum vector $S$.

The row and column sum vectors $R$ and $S$ of a $(0,1)$ - matrix are partitions of the same integer $t$ (its number of 1's). Let $R^{*}=\left(r_{1}^{*}, r_{2}^{*}, \ldots, r_{n}^{*}\right)$ denote the conjugate of $R$ (with trailing 0 's included to get an $n$-tuple). The class $\mathcal{A}\left(R, R^{*}\right)$ is nonempty, and it contains a unique matrix, the perfectly nested matrix $\bar{A}$ with all 1's left justified. Let $R$ and $S$ be proposed row and column sum monotone vectors of a $(0,1)$-matrix that satisfy (1.1). The Gale-Ryser Theorem (see e.g., [4]) asserts that $\mathcal{A}(R, S)$ is nonempty if and only if $S$ is majorized by $R^{*}$ (written $S \preceq R^{*}$ ), that is,

$$
s_{1}+\cdots+s_{k} \leq r_{1}^{*}+\cdots+r_{k}^{*} \quad(k=1,2, \ldots, n)
$$

with equality for $k=n$. If $\mathcal{A}(R, S) \neq \emptyset$, then every matrix in $\mathcal{A}(R, S)$ can be obtained from the perfectly nested matrix $\bar{A}$ with row and column sum vectors $R$ and $R^{*}$, respectively, by shifting 1's in rows to the right. Ryser also proved that given matrices $A$ and $B$ in $\mathcal{A}(R, S)$ then $B$ can be gotten from $A$ by a sequence of interchanges

$$
L_{2}=\left[\begin{array}{ll}
0 & 1 \\
1 & 0
\end{array}\right] \leftrightarrow I_{2}=\left[\begin{array}{ll}
1 & 0 \\
0 & 1
\end{array}\right]
$$

${ }^{*}$ Received by the editors 7 October 2004. Accepted for publication 2 December 2004. Handling Editor: Abraham Berman.

$\dagger$ Department of Mathematics, University of Wisconsin, Madison, WI 53706 (brualdi@math.wisc.edu).

${ }_{\ddagger}^{\ddagger}$ Department of Mathematics Education, Kyungpook University, Taegu 702-701, South Korea (sghwang@knu.ac.kr). Supported by Com² MAC-KOSEF. 
which replace a submatrix equal to $L_{2}$ by $I_{2}$, or the other way around.

There is a well-known order on the symmetric group $S_{n}$ (more generally, on Coxeter groups) of permutations of $\{1,2, \ldots, n\}$ called the Bruhat order, given by:

If $\tau$ and $\pi$ are permutations, then $\pi \leq_{B} \tau$ (in the Bruhat order) provided $\pi$ can be gotten from $\tau$ by a sequence of transformations of the form:

If $a_{i}>a_{j}$, then $a_{1} \cdots a_{i} \cdots a_{j} \cdots a_{n}$ is replaced with $a_{1} \cdots a_{j} \cdots a_{i} \cdots a_{n}$.

Thus if $n=3,123$ is the unique minimal element and 321 is the unique maximal element in the Bruhat order on $S_{3}$.

As usual, the permutations in $S_{n}$ can be identified with the permutation matrices of order $n$, where the permutation $\tau$ corresponds to the permutation matrix $P=\left[p_{i j}\right]$ with $p_{i j}=1$ if and only if $j=\tau(i)$. If $P$ and $Q$ are permutation matrices of order $n$ corresponding to permutations $\tau$ and $\pi$, then we write $P \leq_{B} Q$ whenever $\tau \leq_{B} \pi$. The reduction in the Bruhat order, interpreted for permutation matrices, is that of one-sided interchanges:

$$
L_{2}=\left[\begin{array}{ll}
0 & 1 \\
1 & 0
\end{array}\right] \rightarrow I_{2}=\left[\begin{array}{ll}
1 & 0 \\
0 & 1
\end{array}\right] .
$$

For $n=3$, the minimal permutation (matrix) in the Bruhat order is

$$
I_{3}=\left[\begin{array}{lll}
1 & 0 & 0 \\
0 & 1 & 0 \\
0 & 0 & 1
\end{array}\right]
$$

and the maximal permutation matrix is

$$
D_{3}=\left[\begin{array}{lll}
0 & 0 & 1 \\
0 & 1 & 0 \\
1 & 0 & 0
\end{array}\right] .
$$

There are equivalent ways to define the Bruhat order on $S_{n}$. One is in terms of the Gale order (see e.g., [1]) on subsets of size $k$ of $\{1,2, \ldots, n\}$. Let $k$ be an integer with $1 \leq k \leq n$, and let $X=\left\{a_{1}, a_{2}, \ldots, a_{k}\right\}$ and $Y=\left\{b_{1}, b_{2}, \ldots, b_{k}\right\}$ be subsets of $\{1,2, \ldots, n\}$ of size $k$ where $a_{1}<a_{2}<\cdots<a_{k}$ and $b_{1}<b_{2}<\cdots<b_{k}$. Then in the Gale order, $X \leq_{G} Y$ if and only if $a_{1} \leq b_{1}, a_{2} \leq b_{2}, \ldots, a_{k} \leq b_{k}$. For $\tau=i_{1} i_{2} \ldots i_{n} \in S_{n}$, let $\tau[k]=\left\{i_{1}, i_{2}, \ldots, i_{k}\right\}$. Then it is straightforward to check that, if also $\pi \in S_{n}$, then

$$
\tau \leq_{B} \pi \quad \text { if and only if } \quad \tau[k] \leq_{G} \pi[k] \quad(k=1,2, \ldots, n) .
$$

For an $m$ by $n$ matrix $A=\left[a_{i j}\right]$, let $\Sigma_{A}$ denote the $m$ by $n$ matrix whose $(k, l)$ entry equals

$$
\sigma_{k l}(A)=\sum_{i=1}^{k} \sum_{j=1}^{l} a_{i j} \quad(1 \leq k \leq m ; 1 \leq l \leq n),
$$


the sum of the entries in the leading $k$ by $l$ submatrix of $A$. Using the Gale order, one easily checks that for permutation matrices $P$ and $Q$ of order $n, P \leq{ }_{B} Q$ if and only if $\Sigma_{P} \geq \Sigma_{Q}$, where this latter order is entrywise order.

The Bruhat order on permutation matrices can be extended to the classes $\mathcal{A}(R, S)$. For $A_{1}$ and $A_{2}$ in $\mathcal{A}(R, S)$ we define $A_{1} \leq_{B} A_{2}$ provided, in the entrywise order, $\Sigma_{A_{1}} \geq \Sigma_{A_{2}}$. It is immediate that if $A_{1}$ and $A_{2}$ are matrices in $\mathcal{A}(R, S)$ and $A_{1}$ is obtained from $A_{2}$ by a sequence of one-sided interchanges

$$
L_{2}=\left[\begin{array}{ll}
0 & 1 \\
1 & 0
\end{array}\right] \rightarrow I_{2}=\left[\begin{array}{ll}
1 & 0 \\
0 & 1
\end{array}\right]
$$

then $A_{1} \leq_{B} A_{2}$. This observation gives the following corollary.

Corollary 1.1. Let $A$ be a matrix in $\mathcal{A}(R, S)$ that is minimal in the Bruhat order. Then no submatrix of $A$ equals $L_{2}$.

ExAmple. Let $R=S=(2,2,2,2,2)$. Then

$$
A=\left[\begin{array}{ccccc}
1 & 1 & 0 & 0 & 0 \\
1 & 1 & 0 & 0 & 0 \\
0 & 0 & 1 & 1 & 0 \\
0 & 0 & 1 & 0 & 1 \\
0 & 0 & 0 & 1 & 1
\end{array}\right], \quad \Sigma_{A}=\left[\begin{array}{ccccc}
1 & 2 & 2 & 2 & 2 \\
2 & 4 & 4 & 4 & 4 \\
2 & 4 & 5 & 6 & 6 \\
2 & 4 & 6 & 7 & 8 \\
2 & 4 & 6 & 8 & 10
\end{array}\right]
$$

and

$$
B=\left[\begin{array}{ccccc}
1 & 1 & 0 & 0 & 0 \\
1 & 0 & 1 & 0 & 0 \\
0 & 1 & 1 & 0 & 0 \\
0 & 0 & 0 & 1 & 1 \\
0 & 0 & 0 & 1 & 1
\end{array}\right], \quad \Sigma_{B}=\left[\begin{array}{ccccc}
1 & 2 & 2 & 2 & 2 \\
2 & 3 & 4 & 4 & 4 \\
2 & 4 & 6 & 6 & 6 \\
2 & 4 & 6 & 7 & 8 \\
2 & 4 & 6 & 8 & 10
\end{array}\right]
$$

are both minimal elements of $\mathcal{A}(R, S)$ in the Bruhat order.

Let $A$ be a matrix in $\mathcal{A}(R, S)$ which is minimal in the Bruhat order. Let $A^{c}=J_{m, n}-A$ be the complement of $A$. Here $J_{m, n}$ is the $m$ by $n$ matrix of all 1 's (abbreviated to $J_{n}$ when $m=n$ ), and thus $A^{c}$ has 1 's exactly where $A$ has 0 's. Let $R^{c}$ and $S^{c}$ be, respectively, the row and column sum vectors of $A^{c}$. Since $R$ and $S$ are monotone nonincreasing, $R^{c}$ and $S^{c}$ are monotone nondecreasing. Since $\Sigma_{A^{c}}=\Sigma_{J_{m, n}}-\Sigma_{A}$, it follows that, after reordering rows and columns to get monotone nonincreasing vectors $\widehat{R^{c}}=\left(n-r_{m}, \ldots, n-r_{1}\right)$ and $\widehat{S^{c}}=\left(m-s_{n}, \ldots, m-s_{1}\right)$, the resulting matrix $\widehat{A^{c}}$ is a maximal matrix in the class $\mathcal{A}\left(\widehat{R^{c}}, \widehat{S^{c}}\right)$.

EXAmple. Let $R=S=(2,2,2,2,2)$. Then $\widehat{R^{c}}=\widehat{S^{c}}=(3,3,3,3,3)$. A matrix in $\mathcal{A}\left(\widehat{R^{c}}, \widehat{S^{c}}\right)$ that is minimal in the Bruhat order is the matrix

$$
\left[\begin{array}{lllll}
1 & 1 & 1 & 0 & 0 \\
1 & 1 & 1 & 0 & 0 \\
1 & 0 & 0 & 1 & 1 \\
0 & 1 & 0 & 1 & 1 \\
0 & 0 & 1 & 1 & 1
\end{array}\right]
$$


Thus the matrix

$$
\left[\begin{array}{lllll}
0 & 0 & 0 & 1 & 1 \\
0 & 0 & 0 & 1 & 1 \\
0 & 1 & 1 & 0 & 0 \\
1 & 0 & 1 & 0 & 0 \\
1 & 1 & 0 & 0 & 0
\end{array}\right]
$$

is a matrix in $\mathcal{A}(R, S)$ that is maximal in the Bruhat order.

2. An Algorithm for a Minimal Matrix. In this section we give an algorithm that, starting from the perfectly nested matrix in $\mathcal{A}\left(R, R^{*}\right)$, constructs a matrix in $\mathcal{A}(R, S)$ that is minimal in the Bruhat order. From the above discussion, it follows that we also get an algorithm for constructing a matrix in $\mathcal{A}(R, S)$ that is maximal in the Bruhat order.

\section{Algorithm to Construct a Minimal Matrix in the Bruhat Order}

Let $R=\left(r_{1}, r_{2}, \ldots, r_{m}\right)$ and $S=\left(s_{1}, s_{2}, \ldots, s_{n}\right)$ be monotone nonincreasing positive integral vectors with $S \preceq R^{*}$. Let $\bar{A}$ be the unique matrix in $\mathcal{A}\left(R, R^{*}\right)$.

1. Rewrite $R$ by grouping together its components of equal value:

$$
R=\left(a_{1}, \ldots, a_{1}, a_{2}, \ldots, a_{2}, \ldots, a_{k}, \ldots, a_{k}\right)
$$

where $a_{1}>a_{2}>\cdots>a_{k}$, and the number of $a_{i}$ 's equals $p_{i},(i=1,2, \ldots, k)$.

2. Determine nonnegative integers $x_{1}, x_{2}, \ldots, x_{k}$ satisfying $x_{1}+x_{2}+\cdots+x_{k}=$ $s_{n}$ where $x_{k}, x_{k-1}, \ldots, x_{1}$ are maximized in turn in this order subject to $\left(s_{1}, s_{2}, \ldots, s_{n-1}\right) \preceq R_{1}^{*}$ where $R_{1}=R_{\left(x_{1}, x_{2}, \ldots, x_{k}\right)}$ is the vector

$$
(\underbrace{a_{1}, \ldots, a_{1}, \overbrace{a_{1}-1, \ldots, a_{1}-1}^{x_{1}}}_{p_{1}}, \ldots, \underbrace{a_{k}, \ldots, a_{k} \overbrace{a_{k}-1, \ldots, a_{k}-1}^{x_{k}}}_{p_{k}}) .
$$

3. Shift $s_{n}=x_{1}+x_{2}+\cdots+x_{k}$ 1's to the last column as specified by those rows whose sums have been diminished by 1 : thus the last column consists of $p_{1}-x_{1} 0$ 's followed by $x_{1} 1$ 's, $\ldots, p_{k}-x_{k} 0$ 's followed by $x_{k} 1$ 's.

4. Proceed recursively and return to step 1 , with $R$ replaced with $R_{1}$ and $S$ replaced with $S_{1}=\left(s_{1}, s_{2}, \ldots, s_{n-1}\right)$

ExAmple. Let $R=(\underline{4}, 4,3,3,2,2), S=(4,4,3,3,3,1)$. Then $R^{*}=(6,6,4,2,0,0)$. Starting with the matrix $\bar{A}$ in $\mathcal{A}\left(R, R^{*}\right)$ and applying the algorithm, we get:

$$
\left[\begin{array}{llllll}
1 & 1 & 1 & 1 & 0 & 0 \\
1 & 1 & 1 & 1 & 0 & 0 \\
1 & 1 & 1 & 0 & 0 & 0 \\
1 & 1 & 1 & 0 & 0 & 0 \\
1 & 1 & 0 & 0 & 0 & 0 \\
1 & 1 & 0 & 0 & 0 & 0
\end{array}\right] \rightarrow\left[\begin{array}{llllll}
1 & 1 & 1 & 1 & 0 & 0 \\
1 & 1 & 1 & 1 & 0 & 0 \\
1 & 1 & 1 & 0 & 0 & 0 \\
1 & 1 & 1 & 0 & 0 & 0 \\
1 & 1 & 0 & 0 & 0 & 0 \\
1 & 0 & 0 & 0 & 0 & 1
\end{array}\right] \rightarrow
$$




$$
\left[\begin{array}{llllll}
1 & 1 & 1 & 1 & 0 & 0 \\
1 & 1 & 1 & 1 & 0 & 0 \\
1 & 1 & 1 & 0 & 0 & 0 \\
1 & 1 & 0 & 0 & 1 & 0 \\
1 & 0 & 0 & 0 & 1 & 0 \\
0 & 0 & 0 & 0 & 1 & 1
\end{array}\right] \rightarrow\left[\begin{array}{llllll}
1 & 1 & 1 & 1 & 0 & 0 \\
1 & 1 & 1 & 1 & 0 & 0 \\
1 & 1 & 1 & 0 & 0 & 0 \\
1 & 1 & 0 & 0 & 1 & 0 \\
0 & 0 & 0 & 1 & 1 & 0 \\
0 & 0 & 0 & 0 & 1 & 1
\end{array}\right]
$$

We can stop at this point since no more shifting has to be done. The resulting matrix has no submatrix equal to $L_{2}$, and it is straightforward to verify that it is a minimal matrix in its class $\mathcal{A}(R, S)$.

THEOREM 2.1. Let $R$ and $S$ be positive, monotone vectors such that $\mathcal{A}(R, S)$ is nonempty. Then algorithm I constructs a matrix $A=\left[a_{i j}\right]$ in $\mathcal{A}(R, S)$ that is minimal in the Bruhat order.

Proof. We prove the theorem by induction on $n$. If $n=1$, there is a unique matrix in $\mathcal{A}(R, S)$, and the theorem holds trivially. Assume that $n>1$. Let $R_{1}$ be defined as in the algorithm. Let $P=\left[p_{i j}\right]$ be a matrix in $\mathcal{A}(R, S)$ such that $P \preceq_{B} A$. Let $u=\left(u_{1}, u_{2}, \ldots, u_{m}\right)^{T}$ and $v=\left(v_{1}, v_{2}, \ldots, v_{m}\right)^{T}$ be, respectively, the last columns of $A$ and $P$. First suppose that $u=v$. Then the matrices $A^{\prime}$ and $P^{\prime}$ obtained by deleting the last column of $A$ and $P$, respectively, belong to the same class $\mathcal{A}\left(R^{\prime}, S^{\prime}\right)$, and $P^{\prime} \preceq_{B} A^{\prime}$. Since $A^{\prime}$ is constructed by algorithm I, it now follows from the inductive assumption that $P^{\prime}=A^{\prime}$ and hence $P=A$.

Now suppose that $u \neq v$. We may assume that the last column of $P$ consists of $p_{1}-y_{1}$ 0's followed by $y_{1} 1$ 's, $\ldots, p_{k}-y_{k} 0$ 's followed by $y_{k} 1$ 's where $y_{1}, y_{2}, \ldots, y_{k}$ are nonnegative integers satisfying $y_{1}+y_{2}+\cdots+y_{k}=s_{n}$. Otherwise, the last column of $P$ contains a 1 above a 0 in two rows with equal sums, and $P$ contains a submatrix equal to $L_{2}$. A one-sided interchange then replaces $P$ with $Q$ where $Q \preceq_{B} P \preceq_{B} A$.

The row sum vector $R_{\left(y_{1}, y_{2}, \ldots, y_{k}\right)}$ of the matrix $P^{\prime}$ obtained by deleting the last column of $P$ is nonincreasing. Since $P \in \mathcal{A}(R, S),\left(s_{1}, s_{2}, \ldots, s_{n-1}\right) \preceq R_{\left(y_{1}, y_{2}, \ldots, y_{k}\right)}^{*}$. The choice of $x_{1}, x_{2}, \ldots, x_{k}$ implies that

$$
y_{1}+\cdots+y_{j} \leq x_{1}+\cdots+x_{j} \quad(j=1,2, \ldots, k)
$$

with equality for $j=k$. Let $q$ be the smallest integer such that $u_{q} \neq v_{q}$. Then it follows from (2.1) that $u_{q}=0$ and $v_{q}=1$. We calculate that

$$
\begin{aligned}
\sum_{i=1}^{q} \sum_{j=1}^{n-1} p_{i j} & =r_{1}+\cdots+r_{q}-\sum_{j=1}^{q-1} v_{j}-1 \\
& =r_{1}+\cdots+r_{q}-\sum_{j=1}^{q-1} u_{j}-1 \\
& =r_{1}+\cdots+r_{q}-\sum_{j=1}^{q} u_{j}-1
\end{aligned}
$$




$$
=\sum_{i=1}^{q} \sum_{j=1}^{n-1} a_{i j}-1
$$

contradicting that $P \preceq_{B} A$. The theorem now follows. $\square$

We now consider classes $\mathcal{A}$ with constant row and column sums. Let $k$ be an integer with $1 \leq k \leq n$, let $K=(k, k, \ldots, k)$, the $n$-vector of $k$ 's, and let $R=S=K$. We denote the corresponding class $\mathcal{A}(R, S)$ by $\mathcal{A}(n, k)$. In case $k=1$, this gives the class of permutation matrices of order $n$. Our algorithm for constructing a minimal matrix in $\mathcal{A}(K, K)$ simplifies in this case.

II. Algorithm to Construct a Minimal Matrix in the Bruhat order for $\mathcal{A}(n, k)$

1. Let $n=q k+r$ where $0 \leq r<k$.

2. If $r=0$, then $A=J_{k} \oplus \cdots \oplus J_{k},\left(q J_{k}\right.$ 's $)$ is a minimal matrix.

3. Else, $r \neq 0$.

(a) If $q \geq 2$, let

$$
A=X \oplus J_{k} \oplus \cdots \oplus J_{k},\left(q-1 J_{k} \text { 's, } X \text { has order } k+r\right),
$$

and let $n \leftarrow k+r$.

(b) Else, $q=1$, and let

$$
A=\left[\begin{array}{c|c}
J_{r, k} & O_{k} \\
\hline X & J_{k, r}
\end{array}\right],(X \text { has order } k),
$$

and let $n \leftarrow k$ and $k \leftarrow k-r$.

(c) Proceed recursively with the current values of $n$ and $k$ to determine $X$.

EXAMPLE. Let $n=18$ and $k=11$. The algorithm constructs the following minimal matrix in $\mathcal{A}(K, K)$.

$$
\left[\begin{array}{c|c|c|c}
\multicolumn{3}{|c|}{J_{7,11}} & O_{7} \\
\hline J_{3,4} & O_{3} & O_{7,4} & \\
\hline I_{4} & J_{4,3} & J_{11,7} \\
\hline \multicolumn{2}{|c|}{O_{4,7}} & J_{4} &
\end{array}\right]
$$

Here we first construct (with $18=1 \cdot 11+7$ ),

$$
\left[\begin{array}{c|c}
J_{7,11} & O_{7} \\
\hline X & J_{11,7}
\end{array}\right]
$$

Then to construct the matrix $X$ of order 11 with $k=11-7=4$ (and $11=2 \cdot 4+3$ ), we construct

$$
\left[\begin{array}{c|c}
Y & O_{7,4} \\
\hline O_{4,7} & J_{4}
\end{array}\right] .
$$

Then to construct the matrix $Y$ of order $4+3=7$ with $k=4$ (and $7=1 \cdot 4+3$ ), we construct

$$
\left[\begin{array}{c|c}
J_{3,4} & O_{3} \\
\hline Z & J_{4,3}
\end{array}\right]
$$


Finally, to construct the matrix $Z$ of order 4 with $k=4-3=1($ and $4=4 \cdot 1+0)$, we construct

$$
Z=I_{1} \oplus I_{1} \oplus I_{1} \oplus I_{1}=I_{4} .
$$

3. Minimal Matrices in $\mathcal{A}(n, 2)$ and $\mathcal{A}(n, 3)$. In this section we characterize the minimal matrices in the classes $\mathcal{A}(n, 2)$ and $\mathcal{A}(n, 3)$. Clearly, if $A$ is minimal, so is its transpose $A^{T}$. We first record a useful lemma.

LEMMA 3.1. Let $k$ and $n$ be positive integers with $n \geq k$, and let $A=\left[a_{i j}\right]$ be a matrix in $\mathcal{A}(n, k)$. Assume that $A$ is minimal in the Bruhat order. Let $p$ and $q$ be integers with $1 \leq p<q \leq n$, and let $r$ be an integer with $0 \leq r<n$. If

$$
a_{1 p}+a_{2 p}+\cdots+a_{r p}=a_{1 q}+a_{2 q}+\cdots+a_{r q},
$$

then $\left(a_{r+1, p}, a_{r+1, q}\right) \neq(0,1)$. (If $r=0$, then both sides of (3.1) are interpreted as 0.$)$

Proof. Assume that $(3.1)$ holds and $\left(a_{r+1, p}, a_{r+1, q}\right)=(0,1)$. Since $A$ has $k 1$ 's in each column, there exists an integer $s$ with $r+1<s \leq n$ such that $\left(a_{s p}, a_{s q}\right)=(1,0)$. Hence $A$ has a submatrix of order 2 equal to $L_{2}$, and $A$ cannot be minimal in the Bruhat order.

The minimal matrices in $\mathcal{A}(n, 2)$ are easily determined. Let $F_{n}$ denote the matrix of order $n$ with 0 's in positions $(1, n),(2, n-2), \ldots,(n, 1)$ and 0 's elsewhere.

THEOREM 3.2. Let $n$ be an integer with $n \geq 2$. Then a matrix in $\mathcal{A}(n, 2)$ is a minimal matrix in the Bruhat order if and only if it is the direct sum of matrices equal to $J_{2}$ and $F_{3}$.

Proof. Let $A=\left(a_{i j}\right)$ be a minimal matrix in $\mathcal{A}(n, 2)$. It follows from several applications of Lemma 3.1 (the case $r=0$ ) to $A$ and its transpose that $A$ has the form

$$
\left[\begin{array}{ccccc}
1 & 1 & 0 & \cdots & 0 \\
1 & a_{22} & & & \\
0 & & & & \\
\vdots & & & & \\
0 & & & &
\end{array}\right] .
$$

If $a_{22}=1$, then $A=J_{2} \oplus A^{\prime}$ where $A^{\prime}$ is a minimal matrix in $\mathcal{A}(n-2,2)$. Suppose that $a_{22}=0$. There exists $i, j \geq 3$ such that $a_{2 j}=a_{i 2}=1$. Since $A$ cannot have a submatrix equal to $L_{2}, a_{i j}=1$, and then it follows that $i=j=3$. Hence $A=F_{3} \oplus A^{\prime}$ where $A^{\prime}$ is a minimal matrix in $\mathcal{A}(n-3,2)$. The theorem now follows by induction on $n$. $\square$

Let

$$
V=\left[\begin{array}{lll|ll}
1 & 1 & 1 & 0 & 0 \\
1 & 1 & 1 & 0 & 0 \\
\hline 1 & 0 & 0 & 1 & 1 \\
0 & 1 & 0 & 1 & 1 \\
0 & 0 & 1 & 1 & 1
\end{array}\right]
$$


For $i \geq 1$, let $U_{i}$ be the matrix in $\mathcal{A}(i+6,3)$ of the form

$$
\left[\begin{array}{ccccccccc}
1 & 1 & 1 & 0 & & & \cdots & & \\
1 & 1 & 1 & 0 & & & \cdots & & \\
1 & 1 & 0 & 1 & & & \cdots & & \\
0 & 0 & 1 & 1 & 1 & & & & \\
& & & \ddots & \ddots & \ddots & & & \\
& & & & 1 & 1 & 1 & 0 & 0 \\
& & & & & 1 & 0 & 1 & 1 \\
& & & & & 0 & 1 & 1 & 1 \\
& & & & 0 & 1 & 1 & 1
\end{array}\right] .
$$

Thus

$$
U_{1}=\left[\begin{array}{lllllll}
1 & 1 & 1 & 0 & 0 & 0 & 0 \\
1 & 1 & 1 & 0 & 0 & 0 & 0 \\
1 & 1 & 0 & 1 & 0 & 0 & 0 \\
0 & 0 & 1 & 1 & 1 & 0 & 0 \\
0 & 0 & 0 & 1 & 0 & 1 & 1 \\
0 & 0 & 0 & 0 & 1 & 1 & 1 \\
0 & 0 & 0 & 0 & 1 & 1 & 1
\end{array}\right]
$$

TheOREM 3.3. Let $n$ be an integer with $n \geq 3$. Then a matrix in $\mathcal{A}(n, 3)$ is a minimal matrix in the Bruhat order if and only if it is the direct sum of matrices equal to $J_{3}, F_{4}, V, V^{T}$ and $U_{i}(i \geq 1)$.

Proof. Let $A=\left[a_{i j}\right]$ be a minimal matrix in $\mathcal{A}(n, 3)$. Then $A$ has the form

$$
\left[\begin{array}{cccccc}
1 & 1 & 1 & 0 & \cdots & 0 \\
1 & a_{22} & & & & \\
1 & & & & & \\
0 & & & & & \\
\vdots & & & & & \\
0 & & & & &
\end{array}\right] .
$$

First suppose that $a_{22}=0$. Then Lemma 3.1 implies that $a_{23}=a_{32}=0$. Since each row of $A$ has three 1 's, there exist $l>j>3$ such that $a_{2 j}=a_{2 l}=1$. Since each column of $A$ has three 1's, there exist $k>i>3$ such that $a_{i 2}=a_{k 2}=1$, and there exist $q>p>3$ such that $a_{p 3}=a_{q 3}=1$. Since column $j$ contains only three 1 's, we must have, by Lemma 3.1, that $p=i$ and $q=k$. But then row $i$ has at least four 1 's, a contradiction. Therefore we have $a_{22}=1$.

We now focus on $a_{23}$.

Case I: Assume that $a_{23}=0$. Let the third 1 in row 2 occur in column $j \geq 3$. There exist integers $k>i \geq 3$ such that $a_{k 3}=a_{i 3}=1$. Since $A$ is minimal, we must have $a_{k j}=a_{i j}=1$. If $j>4$, then $a_{24}=0$, contradicting Lemma 3.1. Hence $j=4$. Using Lemma 3.1 and a little thought, we see that $k=i+1$ and $i \in\{3,4\}$. Thus the 
submatrix $A[\{i, i+1\},\{3,4\}]$ at the intersection of rows $i$ and $i+1$ and columns 3 and 4 equals $J_{2}$, and this submatrix intersects row 3 or row 4 . We now consider two subcases according to the value of $a_{32}$.

First suppose that $a_{32}=1$. Since row 3 has only three 1 's, we see that $i=4$, and applying Lemma 3.1 we see that $a_{35}=1$. Thus $A$ has the form

$$
A=\left[\begin{array}{ccccccc}
1 & 1 & 1 & 0 & 0 & \cdots & 0 \\
1 & 1 & 0 & 1 & 0 & \cdots & 0 \\
1 & 1 & 0 & 0 & 1 & \cdots & 0 \\
0 & 0 & 1 & 1 & & \cdots & \\
0 & 0 & 1 & 1 & & \cdots & \\
0 & 0 & 0 & 0 & & & \\
\vdots & \vdots & \vdots & \vdots & & & \\
0 & 0 & 0 & 0 & & &
\end{array}\right] .
$$

Applying Lemma 3.1 to $A^{T}$, we see that $a_{45}=a_{55}=1$. Hence $A=V^{T} \oplus A^{\prime}$ for some $A^{\prime}$.

Now suppose that $a_{32}=0$. Recall that $i \in\{3,4\}$. Suppose that $i=4$. Since each column contains only three 1 's, we have $a_{33}=a_{34}=0$. Applying Lemma 3.1, we get that $a_{35}=a_{36}=1$. Since $A$ cannot have a submatrix equal to $L_{2}$, we conclude that $a_{45}=a_{55}=1$, giving four 1's in row 4 . Therefore we must have $i=3$. Now $A$ has the form

$$
A=\left[\begin{array}{cccc}
1 & 1 & 1 & 0 \\
1 & 1 & 0 & 1 \\
1 & 0 & 1 & 1 \\
0 & a_{42} & 1 & 1
\end{array}\right]
$$

Since $a_{12}+a_{22}+a_{32}=a_{13}+a_{23}+a_{33}$ and $a_{43}=1$, we have, from Lemma 3.1, that $a_{42}=1$, and $A=F_{4} \oplus A^{\prime}$ for some $A^{\prime}$.

Case II: Assume that $a_{23}=1$.

First suppose that $a_{32}=0$, and so by Lemma 3.1, $a_{33}=0$. Since rows 1 and 2 contain only 0 's beyond column 3 , and since row 4 contains three 1's, it again follows from Lemma 3.1 that $a_{34}=a_{35}=1$. Since $a_{i 1}=0$ for all $i \geq 4$, applying Lemma 3.1 to $A^{T}$, we have $a_{42}=1$, and to avoid $L_{2}$, we also have $a_{44}=a_{45}=1$, and so $a_{43}=0$. Since $a_{i 1}=a_{i 2}=0$ for all $i \geq 5$, we have $a_{53}=1$ by Lemma 3.1 applied to $A^{T}$, and using Lemma 3.1 again we see that $a_{54}=a_{55}=1$. Therefore, $A=V \oplus A^{\prime}$ for some matrix $A^{\prime}$.

We now suppose that $a_{32}=1$ so that $A$ begins with the form

$$
\left[\begin{array}{ccc}
1 & 1 & 1 \\
1 & 1 & 1 \\
1 & 1 & a_{33}
\end{array}\right]
$$


If $a_{33}=1$, then $A=J_{3} \oplus A^{\prime}$ for some matrix $A^{\prime}$. Now assume that $a_{33}=0$. By Lemma 3.1 we must have $a_{34}=1$ and, by considering $A^{T}, a_{43}=1$. Hence also $a_{44}=1$. It follows also from Lemma 3.1, using the fact that row 4 contains a 1 in some column $k$ with $k \geq 5$, that $a_{45}=a_{54}=1$. Suppose that $a_{55}=0$. Then $a_{56}=a_{57}=1$ by Lemma 3.1 , and by symmetry, $a_{65}=a_{75}=1$, implying also that $a_{66}=a_{67}=a_{76}=a_{77}=1$. Hence $A$ has the form

$$
A=\left[\begin{array}{lllllll}
1 & 1 & 1 & 0 & 0 & 0 & 0 \\
1 & 1 & 1 & 0 & 0 & 0 & 0 \\
1 & 1 & 0 & 1 & 0 & 0 & 0 \\
0 & 0 & 1 & 1 & 1 & 0 & 0 \\
0 & 0 & 0 & 1 & 0 & 1 & 1 \\
0 & 0 & 0 & 0 & 1 & 1 & 1 \\
0 & 0 & 0 & 0 & 1 & 1 & 1
\end{array}\right]
$$

Therefore, $A=U_{1} \oplus A^{\prime}$ for some matrix $A^{\prime}$. Now suppose that $a_{55}=1$. Then $a_{56}=a_{65}=1$. If $a_{66}=0$, then arguing as above we see that $A=U_{2} \oplus A^{\prime}$ for some matrix $A^{\prime}$. Otherwise we continue and eventually see that $A=U_{i} \oplus A^{\prime}$ for some integer $i$ and matrix $A^{\prime}$.

It would be interesting to characterize all minimal matrices in the Bruhat order for $k \geq 4$ as done for $k=2$ and $k=3$. To do this would require a characterization, for all $k \leq n$, of all minimal matrices in $\mathcal{A}(n, k)$ which cannot be expressed as a nontrivial direct sum. But even for $k=4$, this appears difficult. For example, the following matrices are minimal matrices in $\mathcal{A}(n, 4)$ for an appropriate $n$ that cannot be expressed as a nontrivial direct sum.

$$
\left[\begin{array}{lllllllllllllllll}
1 & 1 & 1 & 1 & & & & & & & & & & & & \\
1 & 1 & 1 & 1 & & & & & & & & & & & & \\
1 & 1 & 1 & 1 & & & & & & & & & & & & \\
1 & 1 & 1 & 0 & 1 & & & & & & & & & & & \\
& & & 1 & 1 & 1 & 1 & 0 & & & & & & & & \\
& & & & 1 & 1 & 1 & 1 & & & & & & & & \\
& & & & 1 & 1 & 1 & 1 & & & & & & & & \\
& & & & 0 & 1 & 1 & 1 & 1 & & & & & & & \\
& & & & & & & 1 & 1 & 1 & 1 & 0 & & & & \\
& & & & & & & & 1 & 1 & 1 & 1 & & & & \\
& & & & & & & & 1 & 1 & 1 & 1 & & & & \\
& & & & & & & & 0 & 1 & 1 & 1 & 1 & & & \\
& & & & & & & & & & & 1 & 0 & 1 & 1 & 1 \\
& & & & & & & & & & & & 1 & 1 & 1 & 1 \\
& & & & & & & & & & & & 1 & 1 & 1 & 1 \\
& & & & & & & & & & & 1 & 1 & 1 & 1
\end{array}\right],
$$




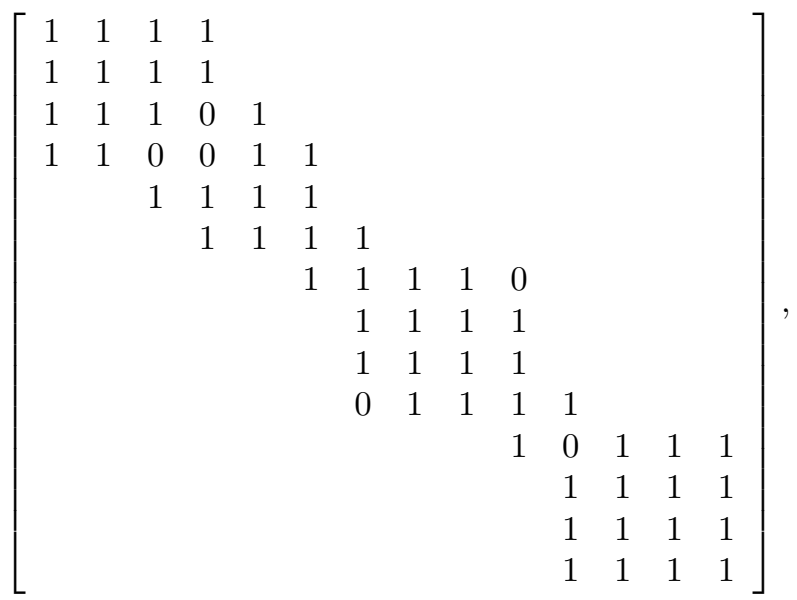

and

$$
\left[\begin{array}{ccccccccccc}
1 & 1 & 1 & 1 & & & & & & & \\
1 & 1 & 1 & 1 & & & & & & & \\
1 & 1 & 1 & 0 & 1 & & & & & & \\
1 & 1 & 0 & 0 & 1 & 1 & & & & & \\
& & 1 & 1 & 1 & 1 & 0 & & & & \\
& & & 1 & 1 & 1 & 1 & & & & \\
& & & & 0 & 1 & 1 & 1 & 1 & & \\
& & & & & & 1 & 1 & 0 & 1 & 1 \\
& & & & & & 1 & 0 & 1 & 1 & 1 \\
& & & & & & & 1 & 1 & 1 & 1 \\
& & & & & & & 1 & 1 & 1 & 1
\end{array}\right] .
$$

In fact, there are many more that can be constructed.

We conclude this note with a conjecture. By Corollary 1.1, a minimal matrix in $\mathcal{A}(R, S)$ has no submatrix equal to $L_{2}$. We conjecture that the converse holds.

Conjecture. A matrix in $\mathcal{A}(R, S)$ that does not have $L_{2}$ as a submatrix is minimal in the Bruhat order on $\mathcal{A}(R, S)$.

\section{REFERENCES}

[1] A.V. Borovik, I.M. Gelfand, and N. White. Coxeter Matroids. Bikhäuser, Boston-Basel-Berlin, 2003.

[2] R.A. Brualdi. Matrices of 0's and 1's with fixed row and column sum vectors. Linear Algebra Appl., 33:159-231, 1980.

[3] R.A. Brualdi. Combinatorial Classes of Matrices, in preparation.

[4] H.J. Ryser. Combinatorial Mathematics. Carus Math. Monograph \# 14, Math. Assoc. of America, Providence, 1964. 